\title{
On the Effect of Stochastic Extra Contribution on Optimal Investment Strategies for Stochastic Salary Under the Affine Interest Rate Model in a DC pension Fund
}

\author{
${ }^{1}$ Bright O. Osu, ${ }^{2}$ Edikan E. Akpanibah and ${ }^{3}$ Njoku K. N. C \\ ${ }^{1}$ Department of Mathematics, Michael Okpara University of Agriculture, Umudike, Nigeria. \\ osu.brjght@mouau.edu.ng \\ ${ }^{2}$ Department of Mathematics and Statistics, Federal University Otuoke, P.M.B 126, Yenagoa, Bayelsa State, Nigeria. \\ edikanakpanibah@gmail.com \\ ${ }^{3}$ Department of Mathematics, Imo State University, P. M. B 2000, Owerri, Nigeria.
}

\begin{abstract}
The essence of this research is to study the optimal investment strategy for a plan contributor in a defined contribution (DC) pension scheme, with stochastic salary and stochastic extra contribution, under the affine interest rate model. We considered the Nigerian Pension Reform Act of 2004 which allow members to contribute an additional proportion of their income into the pension account different from the mandatory contribution. Also we considered investment in cash, zero coupon bond and stock. By using Legendre transformation method and dual theory we obtained the optimal investment strategies for the three investments under the exponential utility function. We observed that the extra contribution has an effect on the optimal investment strategies. The result shows that the pension member will reduce the proportion of his wealth to be invested in cash and increase the proportion to be invested in bond and stock.
\end{abstract}

Keywords: DC Pension fund, Optimal Investment Strategies, Legendre Transform, stochastic extra contribution rate, stochastic salary, exponential utility function.

\section{Introduction}

Recently the practice of DC pension scheme has grown rapidly in most countries since it has provided a convenience plat form for members to involve in it. One of the most attractive features of this scheme is that the member has an idea of what to expect at the time of retirement. Since the member knows that he benefit depend on the various investments which may include investment in one or more of riskless asset (cash), coupon bond, and stock. The need to understand the risk involve and the possible way to minimize the risk has developed in the past years with the study of optimal investment strategies for the investment involved.

$[3,4]$, studied the optimal portfolio problem in a DC pension fund with stochastic interest rate, they assume the interest rate to be of Vasicek model, [5,6], studied the same problem but assume the 
On the Effect of Stochastic Extra Contribution on Optimal Investment Strategies for Stochastic Salary under the Affine

Interest Rate Model in a DC pension fund

interest rate to have an affine structure i.e a model with Vasicek and Cox - Ingeroll - Ross (CIR) model. [3], considered risk incurred from the salary and inflation using vasicek model to determine the optimal allocation strategy. [2], studied optimal investment strategies with stochastic salary under affine interest rate.

In studying the optimal investment strategy, the need for maximization of the expected utility of the terminal wealth is of necessity. Example include, the Constant Relative Risk Aversion (CRRA) utility function, and (or) the Constant Absolute Risk Aversion (CARA) utility function. Some authors use the power utility function which exhibit CRRA see [4-8], while other use exponential utility function which exhibit CARA see [3,9], also some make use of the two see [2,9]. [1], considered an Inflationary market. In their work, the plan member made extra contribution to amortize the pension fund. The CRRA utility function was used to maximize the terminal wealth. This form the basis of our research,

This paper extend the work of [2] by study the optimal investment strategies with stochastic extra contribution rate to analyze the effect of the extra contribution rate on the optimal investment strategies in the various investment. We also applied the Legendre transformation method to obtain the optimal investment strategies for the respective assets.

\section{Preliminaries}

We start with a complete and frictionless financial market that is continuously open over the fixed time interval $[0, T]$, for $T>0$ representing the retirement time of a given shareholder.

Financial Market: We assume that the market is made up of risk free asset (cash) a zero coupon bond and risky asset (stock). Let $(\Omega, F, P)$ be a complete probability space where $\Omega$ is a real space and $P$ is a probability measure, $\left\{W_{1}(t), W_{2}(t): t \geq 0\right\}$ is a standard two dimensional motion such that they orthogonal to each other. $F$ is the filtration and denotes the information generated by the Brownian motion $\left\{W_{1}(t), W_{2}(t)\right\}$.

Risk free Asset (Cash): Let $S_{0}(t)$ denote the price of the risk free asset at time $\mathrm{t}$ and it is modeled as follows

$\frac{d Z(t)}{Z(t)}=r(t) d t$

$r(t)$ represent the short interest rate process which is given by

$d r(t)=(a-b r(t)) d t-\sigma_{r} d W_{1}(t)$,

$\sigma_{r}=\sqrt{p_{1} r(t)+p_{2}}, t \geq 0$,

Where $a, b,(0), p_{1}$, and $p_{2}$ are positive real numbers. If $p_{1}$ (resp., $p_{2}$ ) is equal to zero, we have a special case, as in $[11,12]$. So under these dynamics, the term structure of the short interest rates is affine, which has been studied by [2,5,6,12],

Risky Asset (stock): Let $S_{t}(t)$ denote the risky asset and its dynamics is given based on a continuous time stochastic process at $t \geq 0$ and the price process described as follows

$\frac{d S_{t}(t)}{S_{t}(t)}=r(t) d t+\sigma_{s}\left(d W_{2}(t)+\lambda_{1} d t\right)+n_{1} \sigma_{r}\left(d W_{1}(t)+\lambda_{2} \sigma_{r} d t\right), S_{t}(0)=S_{0}$

with $\lambda_{1}, \lambda_{2}$ (resp., $\sigma_{s}, n_{1}$ ) being constants (resp., positive constants) $[2,5,6]$ 
(Zero Coupon Bond): A zero-coupon bond with maturity $T$, whose price at time $t$ is denoted by $B(t, T), t \geq 0$, and

It dynamics is given by the SDE below $[2,5,6]$

$\frac{d B(t, T),)}{B(t, T),}=r(t) d t+\sigma_{B}(T-t, r(t))\left(d W_{1}(t)+\lambda_{2} \sigma_{r} d t\right), B(T, T)=1$

Where $\sigma_{B}(T-t, r(t))=f(T-t) \sigma_{r}$ and

$f(t)=\frac{2\left(e^{m t}-1\right)}{m-\left(b-p_{1} \lambda_{2}\right)+e^{m t}\left(m+b-p_{1} \lambda_{2}\right)}, m=\sqrt{\left(\left(b-p_{1} \lambda_{2}\right)^{2}+2\right.} p_{1}$

Stochastic Salary: Based on the works of [2-5] we denote the salary at time $t$ by $(t)$ which is described by

$\frac{d L(t)}{L(t)}=\mu_{L}(t, r(t)) d t+n_{2} \sigma_{r} d W_{1}(t)+n_{3} \sigma_{s} d W_{2}(t), L(0)=L_{0}$

where $n_{2}, n_{3}$ are real constants, which are two volatility scale factors measuring how the risk sources of interest rate and stock affect the salary. That is to say, the salary volatility is supposed to a hedgeable volatility whose risk source belongs to the set of the financial market risk sources. This is in accordance with the assumption in [5], but is differs from those of $[3,4]$ who also suggest that the salary was influence by non hedgeable risk source (i.e., non-financial market). Also [2], assume that the instantaneous mean of the salary is such that $\mu_{L}(t, r(t))=r(t)+m_{L}$ where $m_{L}$ is a real constant.

\section{Methodology}

\subsection{Hamilton-Jacobi-Bellman (HJB) equation}

Assume we represent $\pi=\left(\pi_{B}, \pi_{S_{t}}\right)$ as the strategy and we define the utility attained by the contributor from a given state $x$ at time $t$ as

$G_{\pi}(t, r, x)=E_{\pi}[U(X(T)) \mid r(t)=r, X(t)=x]$,

Where $t$ is the time, $S$ is the price of the risky asset and $x$ is the wealth. Our interest here is to find the optimal value function

$G(t, r, x)=\sup _{\pi} G_{\pi}(t, r, x)$

and the optimal strategy $\pi^{*}=\left(\pi_{B}{ }^{*}, \pi_{S_{t}}{ }^{*}\right)$ such that

$G_{\pi^{*}}(t, r, x)=G(t, r, x)$.

\subsection{Legendre Transformation}

The Legendre transform and dual theory help to transform non linear partial differential equation to a linear partial differential equation. 
On the Effect of Stochastic Extra Contribution on Optimal Investment Strategies for Stochastic Salary under the Affine

Theorem 3.1: Let $f: R^{n} \rightarrow R$ be a convex function for $z>0$, define the Legendre transform $N(z)=\max _{x}\{f(x)-z x\}$,

where $N(z)$ is the Legendre dual of $f(x)$. [13]

Since $f(x)$ is convex, from theorem 3.1 we defined the Legendre transform

$$
\widehat{H}(t, r, z)=\sup \{H(t, r, x)-z x \mid 0<x<\infty\} 0<t<T .
$$

where $\widehat{H}$ is the dual of $H$ and $z>0$ is the dual variable of $x$.

The value of $x$ where this optimum is attained is denoted by $h(t, r, z)$, so that

$$
h(t, r, z)=\inf \{x \mid H(t, r, x) \geq z x+\widehat{H}(t, r, z)\} 0<t<T .
$$

The function $h$ and $\widehat{H}$ are closely related and can be refers to as the dual of $H$. These functions are related as follows

$\widehat{H}(t, r, z)=H(t, r, h)-z h$.

Where

$h(t, r, z)=x, H_{x}=z, h=-\widehat{H}_{z}$

At terminal time, we denote

$\widehat{U}(z)=\sup \{U(x)-z x \mid 0<x<\infty\}$,

and

$G(z)=\sup \{x \mid U(x) \geq z x+\widehat{U}(z)\}$.

As a result

$G(z)=\left(U^{\prime}\right)^{-1}(z)$,

where $G$ is the inverse of the marginal utility $U$ and note that $G(T, r, x)=U(x)$

At terminal time $T$, we can define

$h(T, r, z)=\inf _{\mathrm{x}>0}\{x \mid U(x) \geq z x+\widehat{H}(t, r, z)\}$ and $\widehat{H}(t, r, z)=\sup _{\mathrm{x}>0}\{U(x)-z x\}$

so that

$h(T, r, z)=\left(U^{\prime}\right)^{-1}(z)$.

\section{Model Formulation}

We assume that the contributions are continuously paid into the pension fund at the rate of $\left(l_{1} d t+l_{2} d W_{2}(t)\right) L(t)$

where $l_{1}$ is the mandatory rate of contribution and $l_{2}$ is the extra contribution rate which is assume to be stochastic. Let $V(t)$ denote the wealth of pension fund at time $t \in[0, T] . \pi_{B}(t)$ and $\pi_{S}(t)$ represent the proportion of the pension fund invested in the bond and the stock respectively. This implies that the proportion of the pension fund invested in the risk-free asset $\pi_{Z}(t)=1-\pi_{B}(t)-$ $\pi_{S}(t)$.

The dynamics of the pension wealth are given by

$d V(t)=\pi_{Z} V(t) \frac{d Z(t)}{Z(t)}+\pi_{B} V(t) \frac{d B(t, T)}{B(t, T)}+\pi_{S} V(t) \frac{d S(t)}{S(t)}+\left(l_{1} d t+l_{2} d W_{2}(t)\right) L(t)$

Substituting (1), (3) and (4) in to (7) we have

$d V(t)=\left(1-\pi_{B}(t)-\pi_{S}(t)\right) V(t) r(t) d t+\pi_{B} V(t)\left(r(t) d t+\sigma_{B}(T-t, r(t))\left(d W_{1}(t)+\right.\right.$ $\left.\lambda_{2} \sigma_{r} d t\right)+\pi_{S} V(t)\left[r(t) d t+\sigma_{s}\left(d W_{2}(t)+\lambda_{1} d t\right)+n_{1} \sigma_{r}\left(d W_{1}(t)+\lambda_{2} \sigma_{r} d t\right)\right]+\left(l_{1} d t+\right.$ $\left.l_{2} d W_{2}(t)\right) L(t)$

Simplifying (18) we have 
$d V(t)=V(t)\left[r(t)+\lambda_{2} \sigma_{r} \sigma_{B} \pi_{B}+\pi_{S}\left(\lambda_{1} \sigma_{s}+\lambda_{2} \sigma_{r}^{2} n_{1}\right)\right] d t+\left(l_{1} d t+l_{2} d W_{2}(t)\right) L(t)+$

$V(t)\left(\sigma_{B} \pi_{B}+\pi_{S} \sigma_{r} \mathrm{n}_{1}\right) d W_{1}(t)+V(t) \sigma_{S} \pi_{S} d W_{2}(t)$

When it is time for retirement the contributor will be interested in preserving his standard of living and will be interested in his retirement income relative to his predetermine salary. If we consider the contributor salary as a numeraire. Let the relative wealth be defined as follows

$X(t)=\frac{V(t)}{L(t)}$

Applying product rule and Ito's formula to (10) and making use of (6) and (9) we arrive at the following equation

$d X(t)=X\left(r(t)-\mu_{L}+n_{2}^{2}{\sigma_{r}}^{2}+n_{3}{ }^{2} \sigma_{S}{ }^{2}+\sigma_{B} \pi_{B} \sigma_{r}\left(\lambda_{2}-n_{2}\right)+\pi_{S}\left(\lambda_{1} \sigma_{S}+\lambda_{2} \sigma_{r}{ }^{2} n_{1}+\sigma_{S}{ }^{2} n_{3}-\right.\right.$

$\left.n_{1} n_{2}{\sigma_{r}}^{2}\right) d t+\left(l_{1}-\sigma_{S} n_{3} l_{2}\right) d t+X\left(\sigma_{B} \pi_{B}+n_{1} \sigma_{r} \pi_{S}-n_{2} \sigma_{r}\right) d W_{1}(t)+\left(\frac{l_{2}}{X}+X\left(\pi_{S}-n_{3}\right) \sigma_{S}\right) d W_{2}(t)$

$d X(t)=X\left(\theta_{1}+\theta_{2} \pi_{B}+\theta_{3} \pi_{S}\right) d t+\left(l_{1}-\sigma_{S} n_{3} l_{2}\right) d t+X\left(\sigma_{B} \pi_{B}+n_{1} \sigma_{r} \pi_{S}-n_{2} \sigma_{r}\right) d W_{1}(t)+\left(\frac{e_{2}}{X}+\right.$

$\left.X\left(\pi_{S}-n_{3}\right) \sigma_{S}\right) d W_{2}(t)$

$\theta_{1}=r(t)-\mu_{L}+n_{2}^{2}{\sigma_{r}}^{2}+n_{3}^{2} \sigma_{S}^{2}$

$\theta_{2}=\sigma_{B} \sigma_{r}\left(\lambda_{2}-n_{2}\right)$

$\theta_{3}=\lambda_{1} \sigma_{s}+\lambda_{2} \sigma_{r}^{2} n_{1}+\sigma_{S}^{2} n_{3}-n_{1} n_{2} \sigma_{r}^{2}$

The Hamilton-Jacobi-Bellman (HJB) equation associated with the optimization problem is

$H_{t}+(a-b r) H_{r}+\frac{1}{2} \sigma_{r}^{2} H_{r r}+\sup _{\pi}\left\{\left[\left(x\left(\theta_{1}+\pi_{B} \theta_{2}+\pi_{S} \theta_{3}\right)\right)+\left(l_{1}-\sigma_{S} n_{3} l_{2}\right)\right] H_{x}+\frac{1}{2}\left(\frac{l_{2}}{x}+\right.\right.$

$\left.\left.x\left(\pi_{S}-n_{3}\right) \sigma_{S}\right)^{2} H_{x x}+\frac{1}{2} x^{2}\left(\sigma_{B} \pi_{B}+n_{1} \sigma_{r} \pi_{S}-n_{2} \sigma_{r}\right)^{2} H_{x x}-x \sigma_{r}\left(\sigma_{B} \pi_{B}+n_{1} \sigma_{r} \pi_{S}-n_{2} \sigma_{r}\right) H_{r x}\right\}=0$.

where $H_{t}, H_{r}, H_{x}, H_{r x}, H_{r x}$, and $H_{x x}$ denote partial derivatives of first and second orders with respect to time, short interest rate, and relative wealth.

Differentiating (24) with respect to $\pi_{B}$ and $\pi_{S}$, we obtain the first-order maximizing conditions for the optimal strategies $\pi_{B}{ }^{*}$ and $\pi_{S}{ }^{*}$ as

$\theta_{2} H_{x}+x \sigma_{B}\left(\sigma_{B} \pi_{B}+n_{1} \sigma_{r} \pi_{S}-n_{2} \sigma_{r}\right) H_{x x}-\sigma_{r} \sigma_{B} H_{r x}=0$

$\theta_{3} H_{x}+x n_{1} \sigma_{r}\left(\sigma_{B} \pi_{B}+n_{1} \sigma_{r} \pi_{S}-n_{2} \sigma_{r}\right) H_{x x}+x\left(\pi_{S}-n_{3}\right) \sigma_{S}^{2} H_{x x}+$

$\frac{l_{2} \sigma_{S}}{x} H_{x x}-n_{1} \sigma_{r}^{2} H_{r x}=0$

Solving (25) and (26) simultaneously we have

$\pi_{S}{ }^{*}=n_{3}-\frac{l_{2 \sigma_{S}}}{x^{2}}-\left(\frac{\lambda_{1}+n_{3} \sigma_{S}^{2}}{x \sigma_{S}}\right) \frac{H_{x}}{H_{x x}}$

$\pi_{B}^{*}=\frac{\sigma_{r}\left(n_{2}-n_{1} n_{3}+\frac{l_{2} n_{1}}{x^{2} \sigma_{S}}\right)}{\sigma_{B}}+\left(\frac{\theta_{4} \sigma_{r}}{x \sigma_{B}}\right) \frac{G_{x}}{G_{x x}}+\left(\frac{\sigma_{r}}{x \sigma_{B}}\right) \frac{G_{r x}}{G_{x x}}$

$\theta_{4}=\frac{\sigma_{S} n_{2}+n_{1} \lambda_{1}+n_{1} n_{3} \sigma_{S}{ }^{2}-\lambda_{2} \sigma_{S}}{\sigma_{S}}$

Substituting (23), (27), (28), (29) into (17) we have

$$
\begin{aligned}
& H_{t}+(a-b r) H_{r}+\frac{1}{2} \sigma_{r}^{2} H_{r r}+\left(\tau_{0}+\left(\tau_{1}+\tau_{2}\right) x\right) H_{x}+\left(\tau_{3}-\frac{1}{2}\left(\lambda_{2}-n_{2}\right)^{2} \sigma_{r}^{2}\right) \frac{H_{x}^{2}}{H_{x x}}+\left(\lambda_{2}-\right. \\
& \left.n_{2}\right) \sigma_{r}{ }^{2} \frac{H_{x} H_{r x}}{H_{x x}}-\frac{1}{2} \sigma_{r}{ }^{2} \frac{H_{r x}}{H_{x x}}=0 \\
& \tau_{0}=l_{1}-l_{2} \sigma_{S} n_{3}
\end{aligned}
$$


On the Effect of Stochastic Extra Contribution on Optimal Investment Strategies for Stochastic Salary under the Affine

$$
\begin{aligned}
\tau_{1} & =n_{2}{ }^{2}{\sigma_{r}}^{2}+n_{3}{ }^{2} \sigma_{S}{ }^{2}+n_{3} \lambda_{1} \sigma_{S}+\lambda_{2}{\sigma_{r}}^{2} n_{1} n_{3}+{\sigma_{S}}^{2} n_{3}{ }^{2}-n_{1} n_{2} n_{3} \sigma_{r}{ }^{2}-m_{L}-\frac{n_{1} n_{3} \sigma_{r}}{\sigma_{B}} \\
\tau_{2} & =\frac{n_{1} \sigma_{r}}{\sigma_{B}}-\lambda_{1} \sigma_{S}-\lambda_{2} \sigma_{r}{ }^{2} n_{1}-\sigma_{S}{ }^{2} n_{3}+n_{1} n_{2} \sigma_{r}{ }^{2} \\
\tau_{3} & =-\left(\frac{1}{2} \sigma_{S}{ }^{2} n_{3}{ }^{2}+n_{3} \sigma_{S} \lambda_{1}+\frac{1}{2} \lambda_{1}{ }^{2}\right)
\end{aligned}
$$

Differentiating (13) with respect to $t, r$, and $x$ and substituting into (30)

$$
\begin{aligned}
& H_{t}=\widehat{H}_{t}, H_{r}=\widehat{H}_{r}, H_{x}=z, H_{r x}=\frac{-\widehat{H}_{r z}}{\widehat{H}_{z z}}, H_{x x}=\frac{-1}{\widehat{H}_{z z}}, H_{r r}=\widehat{H}_{r r}-\frac{\widehat{H}_{r z}{ }^{2}}{\widehat{H}_{z z}} . \\
& \widehat{H}_{t}+(a-b r) \widehat{H}_{r}+\frac{1}{2} \sigma_{r}{ }^{2} \widehat{H}_{r r}+\left(\tau_{0}+\left(\tau_{1}+\tau_{2}\right) x\right) z-\left(\tau_{3}-\frac{1}{2}\left(\lambda_{2}-n_{2}\right)^{2} \sigma_{r}{ }^{2}\right) z^{2} \widehat{H}_{z z}+ \\
& \left(\lambda_{2}-n_{2}\right) \sigma_{r}{ }^{2} z \widehat{H}_{r z}=0 \\
& \tau_{0}=l_{1}-l_{2} \sigma_{S} n_{3} \\
& \tau_{1}=n_{2}{ }^{2} \sigma_{r}{ }^{2}+2 n_{3}{ }^{2} \sigma_{S}{ }^{2}+n_{3} \lambda_{1} \sigma_{s}+\lambda_{2} \sigma_{r}{ }^{2} n_{1} n_{3}-n_{1} n_{2} n_{3} \sigma_{r}{ }^{2}-m_{L}-\frac{n_{1} n_{3} \sigma_{r}}{\sigma_{B}} \\
& \tau_{2}=\frac{n_{1} \sigma_{r}}{\sigma_{B}}-\lambda_{1} \sigma_{s}-\lambda_{2} \sigma_{r}{ }^{2} n_{1}-\sigma_{S}{ }^{2} n_{3}+n_{1} n_{2} \sigma_{r}{ }^{2} \\
& \tau_{3}=-\left(\frac{1}{2} \sigma_{S}{ }^{2} n_{3}{ }^{2}+n_{3} \sigma_{S} \lambda_{1}+\frac{1}{2} \lambda_{1}{ }^{2}\right)
\end{aligned}
$$

Using $x=h=\widehat{H}_{z}$ and differentiating equation (32) for $\widehat{H}$ with respect to $z$ we obtain a linear PDE in terms of $h$ and its derivatives.

$$
\begin{aligned}
& h_{t}+(a-b r) h_{r}+\frac{1}{2}{\sigma_{r}}^{2} h_{r r}-\tau_{0}-\varphi h-\varphi z h_{z}+\left(\lambda_{2}-n_{2}\right) \sigma_{r}{ }^{2} h_{r}+\left(\lambda_{2}-n_{2}\right) \sigma_{r}{ }^{2} z h_{r z}- \\
& 2\left(\tau_{3}-\frac{1}{2}\left(\lambda_{2}-n_{2}\right)^{2}{\sigma_{r}}^{2}\right) z h_{z}-\left(\tau_{3}-\frac{1}{2}\left(\lambda_{2}-n_{2}\right)^{2}{\sigma_{r}}^{2}\right) z^{2} h_{z z}=0
\end{aligned}
$$

Where

$$
\begin{aligned}
& \varphi=\tau_{1}+\tau_{2} \\
& \tau_{0}=l_{1}-l_{2} \sigma_{S} n_{3} \\
& \tau_{1}=n_{2}{ }^{2} \sigma_{r}{ }^{2}+2 n_{3}{ }^{2} \sigma_{S}{ }^{2}+n_{3} \lambda_{1} \sigma_{S}+\lambda_{2} \sigma_{r}{ }^{2} n_{1} n_{3}-n_{1} n_{2} n_{3} \sigma_{r}{ }^{2}-m_{L}-\frac{n_{1} n_{3} \sigma_{r}}{\sigma_{B}} \\
& \tau_{2}=\frac{n_{1} \sigma_{r}}{\sigma_{B}}-\lambda_{1} \sigma_{S}-\lambda_{2} \sigma_{r}{ }^{2} n_{1}-\sigma_{S}{ }^{2} n_{3}+n_{1} n_{2} \sigma_{r}{ }^{2} \\
& \tau_{3}=-\left(\frac{1}{2} \sigma_{S}{ }^{2} n_{3}{ }^{2}+n_{3} \sigma_{S} \lambda_{1}+\frac{1}{2} \lambda_{1}{ }^{2}\right) \\
& \pi_{Z}{ }^{*}=1-\pi_{B}{ }^{*}-\pi_{S}{ }^{*} \\
& \pi_{S}{ }^{*}=n_{3}-\left(\frac{\lambda_{1}+n_{3} \sigma_{S}{ }^{2}}{h \sigma_{S}}\right) z h_{z}-\frac{l_{2 \sigma_{S}}}{h^{2}} \\
& \pi_{B}{ }^{*}=\frac{\left(n_{2}-n_{1} n_{3}+\frac{l_{2} n_{1}}{\sigma_{S} h^{2}}\right)}{f(T-t)}+\left(\frac{\theta_{4}}{h f(T-t)}\right) z h_{z}-\left(\frac{h_{r}}{h f(T-t)}\right) \\
& \theta_{4}=\frac{\sigma_{S} n_{2}+n_{1} \lambda_{1}+n_{1} n_{3} \sigma_{S}{ }^{2}+\lambda_{2} \sigma_{S}}{\sigma_{S}} \\
& f(t)=\frac{2\left(e^{m t}-1\right)}{m-\left(b-p_{1} \lambda_{2}\right)+e^{m t}\left(m+b-p_{1} \lambda_{2}\right)}, m=\sqrt{\left(\left(b-p_{1} \lambda_{2}\right)^{2}+2\right.} p_{1}
\end{aligned}
$$

We will now solve (33) for $h$ and substitute into (34) and (35) to obtain the optimal investment strategies.

\section{Explicit Solution of the optimal investment strategies for The CRRA Utility Function}

Assume the investor takes a power utility function $U(x)=\frac{x^{q}}{q}, \quad q<1, q \neq 0$ 
The relative risk aversion of an investor with utility described in (36) is constant and (36) is a CRRA utility.

From (16) we have $h(T, r, z)=\left(U^{\prime}\right)^{-1}(z)$ and from (36) we have

$h(T, r, z)=z^{\frac{1}{q-1}}$

We assume a solution to (37) with the following form

Then

$$
h(t, r, z)=v(t, r)\left[z^{\frac{1}{q-1}}\right]+y(t), \quad y(T)=0, \quad v(T, s)=1 .
$$

$$
\begin{aligned}
& h_{t}=v_{t} z^{\frac{1}{q-1}}+y^{\prime}, h_{z}=-\frac{v}{1-q} z^{\left(\frac{1}{q-1}-1\right)}, h_{r z}=-\frac{v_{r}}{1-q} z^{\left(\frac{1}{q-1}-1\right)}, \\
& h_{z z}=\frac{(2-q) v}{(1-q)^{2}} z^{\left(\frac{1}{q-1}-1\right)}, h_{r}=v_{r} z^{\frac{1}{q-1}}, h_{r r}=v_{r r} z^{\frac{1}{q-1}} .
\end{aligned}
$$

Substituting (38) into (33) we have

$\left\{v_{t}+(a-b r) v_{r}-\frac{\left(\lambda_{2}-n_{2}\right) q \sigma_{r}^{2}}{1-q} v_{r}+\frac{1}{2} \sigma_{r}^{2} v_{r r}+\frac{\varphi q v}{1-q}-\frac{q v}{(1-q)^{2}}\left(\tau_{3}-\frac{1}{2}\left(\lambda_{2}-n_{2}\right)^{2} \sigma_{r}^{2}\right)\right\} z^{\frac{1}{q-1}}$

$+y^{I}(t)-\varphi y(t)-\tau_{0}=0$

Splitting (39) we have

$y^{I}(t)-\varphi y(t)-\tau_{0}=0$

$v_{t}+(a-b r) v_{r}-\frac{\left(\lambda_{2}-n_{2}\right) q \sigma_{r}{ }^{2}}{1-q} v_{r}+\frac{1}{2}{\sigma_{r}}^{2} v_{r r}+\frac{\varphi q v}{1-q}-\frac{q v}{(1-q)^{2}}\left(\tau_{3}-\frac{1}{2}\left(\lambda_{2}-n_{2}\right)^{2}{\sigma_{r}}^{2}\right)=0$

Solving (40) with the given boundary condition $y(T)=0$

$y(t)=-\frac{\tau_{0}}{\varphi}\left(1-e^{-\varphi(T-t)}\right)$

To solve (41), we conjecture a solution of the form

$v(t, r)=P(t) e^{Q(t) r} P(T)=1, Q(T)=0$

$v_{r}=P(t) Q(t) e^{N(t) r}, v_{r r}=P(t) Q^{2}(t) e^{N(t) r}$ and $v_{t}=r P(t) Q^{I}(t) e^{Q(t) r}+P^{I}(t) e^{Q(t) r}$

Substituting (43) into (41), we have

$\frac{P^{I}}{P}+\frac{a-\left(\left(\lambda_{2}-n_{2}\right) p_{1}+a\right) q}{1-q} Q+\frac{1}{2} p_{2} Q^{2}+\frac{q\left(\varphi-\tau_{3}-q \varphi\right)}{(1-q)^{2}}+\frac{1}{2} \frac{\left(\lambda_{2}-n_{2}\right)^{2} q p_{2}}{(1-q)^{2}}+r\left(Q^{I}-\frac{b+\left(\left(\lambda_{2}-n_{2}\right) p_{1}-b\right) q}{1-q} Q+\right.$ $\left.\frac{1}{2} p_{1} Q^{2}+\frac{1}{2} \frac{\left(\lambda_{2}-n_{2}\right)^{2} q p_{1}}{(1-q)^{2}}\right)$

Splitting (43) we have

$$
\begin{aligned}
& \frac{P^{I}}{P}+\frac{a-\left(\left(\lambda_{2}-n_{2}\right) p_{1}+a\right) q}{1-q} Q+\frac{1}{2} p_{2} Q^{2}+\frac{q\left(\varphi-\tau_{3}-q \varphi\right)}{(1-q)^{2}}+\frac{1}{2} \frac{\left(\lambda_{2}-n_{2}\right)^{2} q p_{2}}{(1-q)^{2}}=0 \\
& \left.Q^{I}-\frac{b+\left(\left(\lambda_{2}-n_{2}\right) p_{1}-b\right) q}{1-q} Q+\frac{1}{2} p_{1} Q^{2}+\frac{1}{2} \frac{\left(\lambda_{2}-n_{2}\right)^{2} q p_{1}}{(1-q)^{2}}\right)=0 \\
& Q(t)=\frac{d_{1}-d_{2} e^{\frac{1}{2} p_{1}\left(d_{1}-d_{2}\right)(T-t)}}{1-\frac{d_{1}}{d_{2}} e^{\frac{1}{2} p_{1}\left(d_{1}-d_{2}\right)(T-t)}}
\end{aligned}
$$

$P(t)=e^{\left\{\frac{\left(\left(\lambda_{2}-n_{2}\right) p_{1}+a\right) q-a}{1-q} \int Q(t) d t-\frac{1}{2} p_{2} \int Q^{2}(t) d t-\frac{q\left(\varphi-\tau_{3}-q \varphi\right)}{(1-q)^{2}} t+C\right\}} \quad P(T)=1$

Where

$w_{1}=\frac{\left(b+\left(\left(\lambda_{2}-n_{2}\right) p_{1}-b\right) q+\sqrt{\left(b+\left(\left(\lambda_{2}-n_{2}\right) p_{1}-b\right) q\right)^{2}-\left(\lambda_{2}-n_{2}\right)^{2} q p_{1}^{2}}\right)}{(1-q) p_{1}}$ 
On the Effect of Stochastic Extra Contribution on Optimal Investment Strategies for Stochastic Salary under the Affine Interest Rate Model in a DC pension fund

$$
\begin{aligned}
& w_{2}=\frac{\left(b+\left(\left(\lambda_{2}-n_{2}\right) c_{1}-b\right) q+\sqrt{\left(b+\left(\left(\lambda_{2}-n_{2}\right) p_{1}-b\right) q\right)^{2}-\left(\lambda_{2}-n_{2}\right)^{2} q p_{1}^{2}}\right)}{(1-q) p_{1}} \\
& v(r, t)= \\
& \exp \left\{\left(\frac{\left(\left(\lambda_{2}-n_{2}\right) p_{1}+a\right) q-a}{1-q} \int\left(\frac{w_{1}-w_{2} e^{\frac{1}{2} p_{1}\left(w_{1}-w_{2}\right)(T-t)}}{1-\frac{w_{1}}{w_{2}} e^{\frac{1}{2} p_{1}\left(w_{1}-w_{2}\right)(T-t)}}\right) d t-\frac{1}{2} c_{2} \int\left(\frac{w_{1}-w_{2} e^{\frac{1}{2} p_{1}\left(w_{1}-w_{2}\right)(T-t)}}{1-\frac{w_{1}}{w_{2}} e^{\frac{1}{2} p_{1}\left(w_{1}-w_{2}\right)(T-t)}}\right)^{2} d t-\right.\right. \\
& \left.\left.\frac{q\left(\varphi-\tau_{3}-q \varphi\right)}{(1-q)^{2}} t+C\right)+\left(\frac{w_{1}-w_{2} e^{\frac{1}{2} p_{1}\left(w_{1}-w_{2}\right)(T-t)}}{1-\frac{w_{1}}{w_{2}} e^{\frac{1}{2} p_{1}\left(w_{1}-w_{2}\right)(T-t)}}\right) r\right\}
\end{aligned}
$$

Hence the solution of (33) is given as

$h(t, r, z)=$

$\exp \left\{\left(\frac{\left(\left(\lambda_{2}-n_{2}\right) p_{1}+a\right) q-a}{1-q} \int\left(\frac{w_{1}-w_{2} e^{\frac{1}{2} p_{1}\left(w_{1}-w_{2}\right)(T-t)}}{1-\frac{w_{1}}{w_{2}} e^{\frac{1}{2} p_{1}\left(w_{1}-w_{2}\right)(T-t)}}\right) d t-\frac{1}{2} p_{2} \int\left(\frac{w_{1}-w_{2} e^{\frac{1}{2} p_{1}\left(w_{1}-w_{2}\right)(T-t)}}{1-\frac{w_{1}}{w_{2}} e^{\frac{1}{2} p_{1}\left(w_{1}-w_{2}\right)(T-t)}}\right)^{2} d t-\right.\right.$

$\left.\left.\frac{q\left(\varphi-\tau_{3}-q \varphi\right)}{(1-q)^{2}} t+C\right)+\left(\frac{w_{1}-w_{2} e^{\frac{1}{2} p_{1}\left(w_{1}-w_{2}\right)(T-t)}}{1-\frac{w_{1}}{w_{2}} e^{\frac{1}{2} p_{1}\left(w_{1}-w_{2}\right)(T-t)}}\right) r\right\}\left[z^{\frac{1}{q-1}}\right]-\frac{\tau_{0}}{\varphi}\left(1-e^{-\varphi(T-t)}\right)$

\section{Proposition 5.1}

The optimal investment strategies for cash, bond and stock is given as follows

$$
\begin{aligned}
& \pi_{Z}{ }^{*}=1-\pi_{B}{ }^{*}-\pi_{S}{ }^{*} \\
& \pi_{S}^{*}=n_{3}-\frac{l_{2 \sigma_{S}}}{x^{2}}+\left(\frac{\lambda_{1}+n_{3} \sigma_{S}^{2}}{(1-q) \sigma_{S}}\right)+\left(\frac{\lambda_{1}+n_{3} \sigma_{S}^{2}}{(1-q) \sigma_{S}}\right)\left[\frac{\left(l_{1}-l_{2} \sigma_{S} n_{3}\right) l}{x}\left(\frac{\left(1-e^{-\varphi(T-t)}\right)}{\varphi}\right)\right] \\
& \pi_{B}{ }^{*}=\frac{1}{f(T-t)}\left\{\left(n_{2}-n_{1} n_{3}+\frac{l_{2} n_{1}}{\sigma_{S} x^{2}}\right)-\frac{1}{(1-q)}\left(\frac{x \varphi+\left(l_{1}-l_{2} \sigma_{S} n_{3}\right) l}{x \varphi}\right)\left(\theta_{4}+(1-q) Q(t)\right)\left(\left(1-e^{-\varphi(T-t)}\right)\right\}\right. \\
& \theta_{4}=\frac{\sigma_{S} n_{2}+n_{1} \lambda_{1}+n_{1} n_{3} \sigma_{S}^{2}+\lambda_{2} \sigma_{S}}{\sigma_{S}} \\
& h(t, r, z)=v(t, r)\left[z^{\frac{1}{q-1}}\right]+y(t), \\
& y(t)=-\frac{\tau_{0}}{\varphi}\left(1-e^{-\varphi(T-t)}\right) \\
& \varphi=\tau_{1}+\tau_{2} \\
& \tau_{0}=l_{1}-l \sigma_{S} n_{3} \\
& \tau_{1}=n_{2}{ }^{2} \sigma_{r}{ }^{2}+2 n_{3}{ }^{2} \sigma_{S}{ }^{2}+n_{3} \lambda_{1} \sigma_{s}+\lambda_{2} \sigma_{r}{ }^{2} n_{1} n_{3}-n_{1} n_{2} n_{3} \sigma_{r}{ }^{2}-m_{L}-\frac{n_{1} n_{3} \sigma_{r}}{\sigma_{B}} \\
& \tau_{2}=\frac{n_{1} \sigma_{r}}{\sigma_{B}}-\lambda_{1} \sigma_{s}-\lambda_{2} \sigma_{r}^{2} n_{1}-\sigma_{S}^{2} n_{3}+n_{1} n_{2} \sigma_{r}^{2} \\
& \tau_{3}=-\left(\frac{1}{2} \sigma_{S}^{2} n_{3}^{2}+n_{3} \sigma_{s} \lambda_{1}+\frac{1}{2} \lambda_{1}^{2}\right) \\
& h_{r}=v_{r} z^{\frac{1}{q-1}} \text {, } \\
& h_{z}=-\frac{v}{1-q} z^{\left(\frac{1}{q-1}-1\right)} \\
& v(t, r)=P(t) e^{Q(t) r} \\
& v_{r}=P(t) Q(t) e^{Q(t) r}
\end{aligned}
$$




$$
\begin{aligned}
& Q(t)=\frac{w_{1}-w_{2} e^{\frac{1}{2} p_{1}\left(w_{1}-w_{2}\right)(T-t)}}{1-\frac{w_{1}}{w_{2}} e^{\frac{1}{2} p_{1}\left(w_{1}-w_{2}\right)(T-t)}} \\
& P(t)=e^{\left\{\frac{\left(\left(\lambda_{2}-n_{2}\right) p_{1}+a\right) q-a}{1-q} \int Q(t) d t-\frac{1}{2} p_{2} \int Q^{2}(t) d t-\frac{q\left(\varphi-\tau_{3}-q \varphi\right)}{(1-q)^{2}} t+C\right\}}
\end{aligned}
$$

Where

$$
\begin{aligned}
& w_{1}=\frac{\left(b+\left(\left(\lambda_{2}-n_{2}\right) p_{1}-b\right) q+\sqrt{\left(b+\left(\left(\lambda_{2}-n_{2}\right) p_{1}-b\right) q\right)^{2}-\left(\lambda_{2}-n_{2}\right)^{2} q p_{1}^{2}}\right)}{(1-q) p_{1}} \\
& w_{2}=\frac{\left(b+\left(\left(\lambda_{2}-n_{2}\right) c_{1}-b\right) q+\sqrt{\left(b+\left(\left(\lambda_{2}-n_{2}\right) p_{1}-b\right) q\right)^{2}-\left(\lambda_{2}-n_{2}\right)^{2} q p_{1}^{2}}\right)}{(1-q) p_{1}} \\
& v(r, t)= \\
& \exp \left\{\left(\frac{\left(\left(\lambda_{2}-n_{2}\right) p_{1}+a\right) q-a}{1-q} \int\left(\frac{w_{1}-w_{2} e^{\frac{1}{2} p_{1}\left(w_{1}-w_{2}\right)(T-t)}}{1-\frac{w_{1}}{w_{2}} e^{\frac{1}{2} p_{1}\left(w_{1}-w_{2}\right)(T-t)}}\right) d t-\frac{1}{2} p_{2} \int\left(\frac{w_{1}-w_{2} e^{\frac{1}{2} p_{1}\left(w_{1}-w_{2}\right)(T-t)}}{1-\frac{w_{1}}{w_{2}} e^{\frac{1}{2} p_{1}\left(w_{1}-w_{2}\right)(T-t)}}\right)^{2} d t-\right.\right. \\
& \left.\left.\frac{q\left(\varphi-\tau_{3}-q \varphi\right)}{(1-q)^{2}} t+C\right)+\left(\frac{w_{1}-w_{2} e^{\frac{1}{2} p_{1}\left(w_{1}-w_{2}\right)(T-t)}}{1-\frac{w_{1}}{w_{2}} e^{\frac{1}{2} p_{1}\left(w_{1}-w_{2}\right)(T-t)}}\right) r\right\} \\
& f(t)=\frac{2\left(e^{m t}-1\right)}{m-\left(b-p_{1} \lambda_{2}\right)+e^{m t}\left(m+b-p_{1} \lambda_{2}\right)}, m=\sqrt{\left(\left(b-p_{1} \lambda_{2}\right)^{2}+2\right.} p_{1}
\end{aligned}
$$

\section{Discussion and Conclusion}

\section{Discussion}

\section{Remark 6.1}

Suppose $n_{2}=n_{3}=0, q \rightarrow 0, l=1$, then the salary is not stochastic and the optimal investment is given as

$$
\begin{aligned}
& \pi_{S}{ }^{*}=\left(\frac{\lambda_{1}}{\sigma_{S}}\right)-\frac{l_{2 \sigma_{S}}}{x^{2}}+\left(\frac{\lambda_{1}}{\sigma_{S}}\right)\left[\frac{\left(l_{1}-l_{2} \sigma_{S} n_{3}\right) l}{x}\left(\frac{\left(1-e^{-\varphi(T-t)}\right)}{\varphi}\right)\right] \\
& \pi_{B}{ }^{*}=\frac{1}{f(T-t)}\left\{\left(\frac{\lambda_{2} \sigma_{S}-n_{1} \lambda_{1}}{\sigma_{S}}\right)\left(\frac{\left.x \varphi+l_{1}\right)}{x \varphi}\right)\left(\left(1-e^{-\varphi(T-t)}\right)\right\}\right.
\end{aligned}
$$

Also assume there is no extra contribution i.e , $l_{2}=0$ we have

$$
\begin{aligned}
& \pi_{S_{1}}{ }^{*}=\left(\frac{\lambda_{1}}{\sigma_{S}}\right)+\left(\frac{\lambda_{1}}{\sigma_{S}}\right)\left[\frac{l_{1}}{x}\left(\frac{\left(1-e^{-\varphi(T-t)}\right)}{\varphi}\right)\right] \\
& \pi_{B_{1}}{ }^{*}=\frac{1}{f(T-t)}\left\{\left(\frac{\lambda_{2} \sigma_{S}-n_{1} \lambda_{1}}{\sigma_{S}}\right)\left(\frac{x \varphi+l_{1}}{x \varphi}\right)\left(\left(1-e^{-\varphi(T-t)}\right)\right\}\right.
\end{aligned}
$$

\section{Proposition 6.1}

Suppose $\pi_{S_{1}}{ }^{*}<\pi_{S}{ }^{*}$ and $\pi_{B_{1}}{ }^{*}<\pi_{B}{ }^{*}$ then $\pi_{Z_{1}}{ }^{*}>\pi_{Z}{ }^{*}$ 
On the Effect of Stochastic Extra Contribution on Optimal Investment Strategies for Stochastic Salary under the Affine Interest Rate Model in a DC pension fund

Proof

Since $\pi_{S_{1}}{ }^{*}<\pi_{S}{ }^{*}$ and $\pi_{B_{1}}{ }^{*}<\pi_{B}{ }^{*}$ then,

$\pi_{S_{1}}{ }^{*}+\pi_{B_{1}}{ }^{*}<\pi_{S}{ }^{*}+\pi_{B}{ }^{*}$

$1-\left(\pi_{S_{1}}{ }^{*}+\pi_{B_{1}}{ }^{*}\right)>1-\left(\pi_{S}{ }^{*}+\pi_{B}{ }^{*}\right)$

$1-\pi_{S_{1}}{ }^{*}-\pi_{B_{1}}{ }^{*}>1-\pi_{S}{ }^{*}-\pi_{B}{ }^{*}$

But $\pi_{Z_{1}}{ }^{*}=1-\pi_{S_{1}}{ }^{*}-\pi_{B_{1}}{ }^{*}$ and $\pi_{Z}{ }^{*}=1-\pi_{S}{ }^{*}-\pi_{B}{ }^{*}$, therefore

$\pi_{Z_{1}}{ }^{*}>\pi_{Z}^{*}$

\section{Remark 6.2}

Suppose $l_{2}=0$ then the strategies in proposition (5.1) is without the extra contribution hence the required strategies are as given below

$$
\begin{aligned}
& \pi_{S_{2}}{ }^{*}=n_{3}+\left(\frac{\lambda_{1}+n_{3} \sigma_{S}^{2}}{(1-q) \sigma_{S}}\right)+\left(\frac{\lambda_{1}+n_{3} \sigma_{S}^{2}}{(1-q) \sigma_{S}}\right)\left[\frac{\left(l_{1}\right) l}{x}\left(\frac{\left(1-e^{-\varphi(T-t)}\right)}{\varphi}\right)\right] \\
& \pi_{B_{2}}{ }^{*}=\frac{1}{f(T-t)}\left\{\left(n_{2}-n_{1} n_{3}\right)-\frac{1}{(1-q)}\left(\frac{x \varphi+\left(l_{1}\right) l}{x \varphi}\right)\left(\theta_{4}+(1-q) Q(t)\right)\left(\left(1-e^{-\varphi(T-t)}\right)\right\}\right.
\end{aligned}
$$

The result above is similar to that in [2]

\section{Case 1}

If $n_{3}>0, \lambda_{1}>0, \sigma_{S}>0,0<q<1, \varphi>0, l>0, l_{2}=0$ then $\pi_{S_{2}}{ }^{*}<\pi_{S}{ }^{*}$

\section{Case 2}

If $\left(n_{2}-n_{1} n_{3}\right)>0, f(T-t)>0, \lambda_{1}>0, \sigma_{S}>0,0<q<1, \varphi>0, l>0, Q(t)>0, l_{2}=0$

then $\pi_{B_{2}}{ }^{*}<\pi_{B}{ }^{*}$

\section{Case 3}

If $\left(n_{2}-n_{1} n_{3}\right)<0, f(T-t)>0, \lambda_{1}>0, \sigma_{S}>0,0<q<1, \varphi>0, l>0, Q(t)>0, l_{2}=0$

then $\pi_{\boldsymbol{B}_{2}}{ }^{*}>\pi_{B}{ }^{*}$

\section{Proposition 6.2}

Suppose $\pi_{S_{2}}{ }^{*}<\pi_{S}{ }^{*}$ and $\pi_{B_{2}}{ }^{*}<\pi_{B}{ }^{*}$ then $\pi_{Z_{2}}{ }^{*}>\pi_{Z}{ }^{*}$

Proof

Since $\pi_{S_{2}}{ }^{*}<\pi_{S}{ }^{*}$ and $\pi_{B_{2}}{ }^{*}<\pi_{B}{ }^{*}$ then,

$$
\pi_{S_{2}}{ }^{*}+\pi_{B_{2}}{ }^{*}<\pi_{S}{ }^{*}+\pi_{B}{ }^{*}
$$


$1-\left(\pi_{S_{2}}{ }^{*}+\pi_{B_{2}}{ }^{*}\right)>1-\left(\pi_{S}{ }^{*}+\pi_{B}{ }^{*}\right)$

$1-\pi_{S_{2}}{ }^{*}-\pi_{B_{2}}{ }^{*}>1-\pi_{S}{ }^{*}-\pi_{B}{ }^{*}$

But $\pi_{Z_{2}}{ }^{*}=1-\pi_{S_{2}}{ }^{*}-\pi_{B_{2}}{ }^{*}$ and $\pi_{Z}{ }^{*}=1-\pi_{S}{ }^{*}-\pi_{B}{ }^{*}$, therefore

$\pi_{Z_{2}}{ }^{*}>\pi_{Z}^{*}$

Proposition 6.1, shows that with non stochastic salary, the optimal investment strategies for bond and stock increases with extra contribution while that of cash decreases with extra contribution.

Similarly proposition 6.2 , shows that with stochastic salary, the optimal investment strategies for bond and stock increases with extra contribution while that of cash decreases with extra contribution. In general we observed that extra contribution to the pension fund has an effect on the optimal investment strategies in cash, bond and stock. The analysis shows that the plan member will increase the proportion of his wealth to be invested in bond and stock and will reduce the proportion to be invested in cash.

\section{Conclusion}

We studied optimal investment strategy for a plan contributor in a defined pension scheme, with stochastic salary and stochastic extra contributions, under the affine interest rate model. We took into consideration the Nigerian Pension Reform Act of 2004 which allow members to contribute another proportion of their income into the pension account different from the compulsory contribution. We also considered three different investment which include cash, zero coupon bond) and the stock. By using Legendre transformation method and dual theory we obtained the optimal investment strategies for the three investments under the exponential utility function. We observed that extra contribution to the pension fund has an effect on the optimal investment strategies. The result shows that the pension member will increase the proportion of his wealth to be invested in bond and stock while reducing the proportion to be invested in cash.

\section{References}

[1] Othusitse Basimanebotlhe and Xiaoping Xue, (2015), Stochastic Optimal Investment under Inflamatory Market with Minimum Guarantee for DC Pension Plans, Journal of Mathematics, 7.

[2] Chubing Z. and Ximing R. (2013), Optimal investment strategies for DC pension with stochastic salary under affine interest rate model. Hindawi Publishing Corporation http://dx.doi.org/10.1155/2013/297875

[3] Battocchio P. and .Menoncin F. (2004), Optimal pension management in a stochastic framework, Insurance, 34, 79-95.

[4] Cairns A. J. G, Blake D. and Dowd K. (2006), Stochastic lifestyling: optimal dynamic asset allocation for defined contribution pension plans, Journal of Economic Dynamics \& Control, 30, 
On the Effect of Stochastic Extra Contribution on Optimal Investment Strategies for Stochastic Salary under the Affine

Interest Rate Model in a DC pension fund

843-877

[5] Deelstra, G., Grasselli, M.. and. Koehl, P.F. (2003), Optimal investment strategies in the presence of a minimum guarantee, Insurance, 33, 189-207

[6] Gao J., (2008), Stochastic optimal control of DC pension funds, Insurance, vol. 42, 1159-1164..

[7] Boulier J. F. Huang S., and Taillard G (2001), Optimal management under stochastic interest rates: the case of a protected defined contribution pension fund, Insurance, 28, 173-189.

[8] Xiao J., Hong Z., and Qin C, (2007), The constant elasticity of variance(CEV) model and the Legendre transform-dual solution for annuity contracts, Insurance, 40, 302-310

[9] Gao, J (2009), Optimal portfolios for DC pension plans under a CEV model, Insurance, Mathematics and Economics 44, 479-490

[10]Vasicek O. (1977), An equilibrium characterization of the term structure, Journal of Financial Economics, 5, 177-188

[11]Cox J. C., Ingersoll J. E., and Ross S. A.(1985), A theory of the term structure of interest rates, Econometrica, 53, 385-407.

[12]Duffie D. and Kan R, (1996), A yield-factor model of interest rates, "Mathematical Finance, 6, 379406.

[13]Jonsson M. and Sircar R. (2002), Optimal investment problems and Volatility homogenization approximations, in Modern Methods in Scientific Computing and Applications, 75, 255-281, Springer, Berlin, Germany. 